The book is an outstanding achievement, written by one of the central participants in the modern development of biology. The presentation is clear, authoritative and vivid. The colourful yet concise style is illustrated by the following headings: "Avery's Bombshell" (referring to the discovery that nucleic acids can carry information); "The Twenty-five Year Loneliness of the Protein Crystallographers" (referring to the long lag period before X-ray crystallography yielded major results); "Much More Must be Learned about Ribosomes" (referring to the lack of information on the basic properties of their structure). The book is further enlivened by excellent diagrammatic illustrations. The account is well balanced; achievements as well as gaps in our knowledge are recorded. The approach is altogether highly original. This makes the book profitable not only for the beginner but also for expert biologists of all descriptions including biochemists and geneticists. It was produced within six months and is therefore as up to date as is possible nowadays.

H. A. KreBs

\section{REFLEX ACTIVITY AND THE CENTRAL NERVOUS SYSTEM}

\section{Compensatory Adaptations, Reflex Activity and the Brain}

By Ezras A. Asratian. Scientific and Translation Editor, Prof. Samuel A. Corson. (International Series of Monographs on Cerebrovisceral and Behavioural Physiology and Conditioned Reflexes, Vol. 1.) Pp. xv + 194. (London and New York: Pergamon Press Ltd., 1965.) 80s. net.

\section{7}

HE basic object of Compensatory Adaptations, Reflex Activity and the Brain is to extend the interpretation of Pavlov's work in an attempt to explain reactions in the central nervous system as arising out of conditioned and unconditioned reflexes. Thus, among other subjects, spinal shock, restoration of activity after damage to the spinal cord or the brain, and certain metabolic disturbances zssociated with central nervous exclusion are considered. Conditioned reflexes are suggested to be not restricted to individual neurological levels, but to have a number of associated extensions to the medulla, mid-brain and the cerebral cortex, together with common afferent and efferent pathways.

There are six chapters in the volume, the first four of which are based on a series of lectures given in British universities during 1957-58, but intimate reference to each chapter is not necessary in this review, for the subject matter is not restricted solely to individual sections.

Spinal shock is the subject of the first chapter, the mechanism of its causation affording the main topic. The evidence proclaimed supports Sherrington's views with some qualification. Related changes in the medulle and the parietal cortex are described, their recognition being the outcome of more modern forms of investigation, for example, electro-encephalography.

The use of terms such as 'irritation' and 'stimulation' rather suggests active factors in related causes of reactionary phenomena, as against a negation of function in the form of release of activities of other structures, where inhibition or suppression reigned beforehand.

The claim that the intensity of spinal shock shows some tendency to vary in accordance with the segment of cord damaged is noteworthy; for example, a greater degree of shock is induced by posterior hemisection than lateral hemisection of the cord. It would appear that proprioceptive disorganization has more bearing than the inter ruption in conduction of other afferent stimuli.

Compensatory mechanisms in the return of activity following disablement of experimental dogs would not necessarily indicate true recovery, but a process of adaptation. This seems a more likely explanation of the intensification of their positive conditioned reflexes and a decline of their inhibitory conditioned reflexes (p. 61). Enforoing an animal to apply a reconditioned structure, for example, the arm of the monkey (p. 63), surely is to be expected to expedite return of available activity within the $\operatorname{limb}$, when the opposite limb has been put out of action by restriative measures such as splinting or bandaging, or by amputation. Return of movement is recorded in animals where the following experiments have been undertaken: ambulation, where the forelimb on one side and hind limb on the opposite side have been amputated; cross anastomosis of vagus and median nerves; lateral hemisection of the spinal cord; dorsal hemisection of the spinal cord; longitudinal section of the cord. Moreover, recovery of mobility is abolished should tho animal be subjected later to removal of the cerebris cortex or decerebration.

To record that the inability of activity to appear in animals subjected to cortical excision or decerebration could in any way be an interference with reflex action is a most restricted attitude. Generalized loss of cerebral cortex, in terms of cortical functions, is so disabling that a restricted aspect cannot be determined on this premise.

It is noted that attention to endocrine dysfunction is given some consideration, for not only is this mentioned but illustrations of affected glands occur. Changes in the ovary, pituitary and thyroid are to be seen. Yet the endocrine disorders arising out of these effects are not discussed. The introduction of the destruction of the whole cortex as a means of supporting the hypothesis propounded is a strange dismissal of the many functions assignable to that part of the brain.

The work is certainly worthy of perusal, though the English demands a most careful analysis, for often the sentences are too long. At a price of $80 s$., however, I would not recommend it for other than the most objective attention.

HARVEY JACKSON

\section{THE LIFE OF FISHES}

\section{The Life of Fishes}

By N. B. Marshall. (The Weidenfeld and Nicolson Natural History.) Pp. $402+43$ plates. (London: Weidenfeld and Nicolson, 1965.) 63s. net.

THE Editor of Nature invites reviewers to suggest a separate title for their reviews, but in the case of The Life of Fishes a more apt title than that of the book itself would be difficult to find. Perhaps it would be more accurate to refer to the "Lives of Fishes", for one result of reading this book is to be impressed by the diverse ways in which fishes have become adapted to deal with the manifold facets of life in water, of parcelling out the environment among themselves, and of the ways in which these animals have become masters of their environment.

The scope of this book is immense, even though the author has limited himself to the short and medium timescales in the lives of fishes. As if this was not enough, however, Mr. Marshall also treats the reader to a masterly review of the long time-scale as seen in the results of evolution manifest in the morphological diversity of living fishes. His final chapter, devoted to a succinct account of the classes and orders of fishes, will be highly valued by students who would otherwise have to seek widely for their information.

But the essence of the book is its concern with fishes in their environment, and not least of all with interactions between the fishes, as mates, as food or even to form near 'super-organisms'. Since his approach is a dynamic one, the author has reduced, so far as possible (and very successfully), the purely anatomical elements necessary to understand the physiological, behavioural and ecological specializations of fishes. To a large extent this has been zchieved by the use of clear figures and diagrams.

The first, and largest, section of the book deals with the day-to-day existence of adult fishes. It gives particular emphasis to basic life processes, and to the prob- 